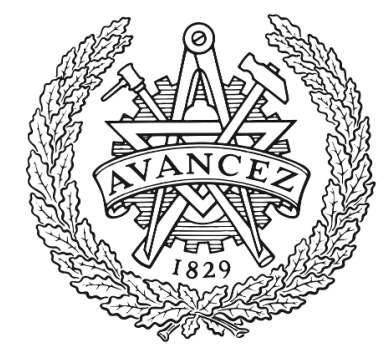

\title{
CHALMERS
}

UNIVERSITY OF TECHNOLOGY

\section{Beam pattern measurement on offset Gregorian reflector mounted with a wideband room temperature receiver for the Square Kilometre Array}

Downloaded from: https://research.chalmers.se, 2023-04-26 09:54 UTC

Citation for the original published paper (version of record):

Flygare, J., Billade, B., Dahlgren, M. et al (2018). Beam pattern measurement on offset Gregorian reflector mounted with a wideband room temperature receiver for the Square Kilometre Array. 2018 IEEE International Symposium on Antennas and Propagation \& USNC/URSI National Radio Science Meeting (APSURSI2018), APSURSI 2018: 1759-1760. http://dx.doi.org/10.1109/APUSNCURSINRSM.2018.8608627

N.B. When citing this work, cite the original published paper. 


\section{Beam pattern measurement on offset Gregorian reflector mounted with a wideband room temperature receiver for the Square Kilometre Array}

\author{
Jonas Flygare, Bhushan Billade, Magnus Dahlgren, \\ Miroslav Pantaleev, Jens Dahlström, Bo Wästberg \\ (Onsala Space Observatory): Dept. of Space, Earth and Environment \\ Chalmers University of Technology \\ SE-43992-Onsala, Sweden \\ jonas.flygare@chalmers.se
}

\author{
Gary Hovey, Richard Hellyer, Rob Messing, \\ Bruce Veidt, Gordon Lacy, Mohammad Islam \\ (DRAO): Herzberg Astronomy and Astrophysics \\ NRC-CNRC \\ Penticton, British Columbia V2A 6J9, Canada \\ gary.hovey@nrc-cnrc.gc.ca
}

\begin{abstract}
We present measured on-dish beam patterns for a room temperature spline-profile quad-ridge flared horn (QRFH) feed designed for the Square Kilometre Array (SKA) Band 1 covering 350-1050 MHz. The feed and LNA package has been mounted on the offset Gregorian SKA precursor prototype telescope, Dish Verification Antenna 1 (DVA-1) located at the Dominion Radio Astrophysical Observatory (DRAO) near Penticton, British Columbia, Canada. Telescope beam pattern cuts are measured by sweeping over the sun and adding corrections for the solar disk size, elevation and aperture efficiency. Sensitivity calculated with measured receiver noise show good agreement with the predicted performance.
\end{abstract}

\section{INTRODUCTION}

The Square Kilometre Array (SKA) is an international collaboration to build the world's largest and most sensitivity radio telescope array. The SKA singel-pixel feed programme aims to cover $0.35-13.8 \mathrm{GHz}$ with five different frequency bands through five separate feed packages mounted on each of the offset Gregorian $15 \mathrm{~m}$ reflector telescopes. The splined Quad-Ridge Flared Horn (QRFH) feed design for the lowest frequency band $350-1050 \mathrm{MHz}$ [1] defined as Band 1 has due to recent development in Low Noise Amplifiers (LNA) [2] been transferred from a cryogenic to a completely room temperature system [3] with comparable noise performance.

In this paper we present telescope beam pattern measurements using a completely room temperature receiver developed at Onsala Space Observatory for $350-1050 \mathrm{MHz}$. The system was installed on the offset Gregorian SKA precursor prototype, Dish Verification Antenna 1 (DVA-1), at the Dominion Radio Astrophysical Observatory (DRAO) near Penticton, British Columbia, Canada. Beam pattern cuts were measured using the sun as source and the results show good agreement with simulation. Measurement of the receiver noise temperature gives confirmation of the feed system feasibility.

\section{System PeRformance}

The 3:1 QRFH feed design was designed with splinedefined profiles and optimized for high $A_{\text {eff }} / T_{\text {sys }}$ (sensitivity) on the SKA reflector optics, which consists of a $15 \mathrm{~m}$ offset

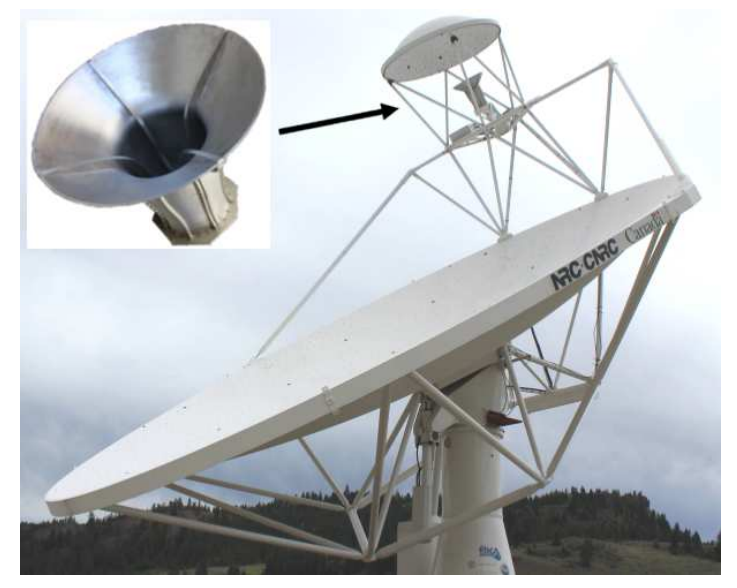

Fig. 1. QRFH (top left) installed on DVA-1, seen just below the sub-reflector.

Gregorian dish with a $5 \mathrm{~m}$ sub-reflector that has a half subtended angle of $\theta_{0}=58^{\circ}$. $A_{\text {eff }}$ is the effective reflector area and $T_{\text {sys }}=T_{\mathrm{a}}+T_{\text {rec }}$ is the system noise temperature where $T_{\mathrm{a}}$ and $T_{\text {rec }}$ are the antenna and receiver noise temperatures respectively. To achieve high sensitivity a reasonable trade-off between spill-over and illumination efficiency is required. The feed prototype shows measured input reflection $S_{11}<-10$ $\mathrm{dB}$ and $T_{\text {rec }}<20 \mathrm{~K}$ (Y-factor tests). The room temperature LNAs, designed by Low Noise Factory (LNF) in Sweden, are integrated inside the horn ridges and directly connected to the feeding pins. In Fig. 1 the QRFH can be seen mounted on the DVA-1, which compared to the SKA reflector has a slightly smaller half subtended angle of $\theta_{0}=55^{\circ}$ and a smaller subreflector without a spill-over shield. The feed aperture efficiency $\eta_{\mathrm{a}}$ simulated on DVA-1 is above $60 \%$ across the band with an average of $71 \%$, see Fig. 2. At the low end of the band there is over illumination of the dish due to the smaller half subtended angle of DVA-1. This also increases the spill-over noise contribution from the ground. We estimate the receiver performance with a system simulator that combines GRASP physical optics (PO) and physical theory of 
diffraction (PTD) [4]. The $A_{\text {eff }} / T_{\text {sys }}$ is given by a full-sphere integration of the sky and ground noise temperatures weighted with the telescope beam pattern. Below $500 \mathrm{MHz}$, the sky noise temperature is several times larger than at the high end of the band, which increases $T_{\mathrm{a}}$. The estimated sensitivity for the feed on DVA-1, Fig. 2 averages $2.91 \mathrm{~m}^{2} / \mathrm{K}$ over the band for zenith angle $\left|\theta_{p}\right|=60^{\circ}$ and $3.54 \mathrm{~m}^{2} / \mathrm{K}$ over $650-1050$ $\mathrm{MHz}$. There is good agreement in sensitivity calculated with estimated and measured $T_{r e c}$. Only frequencies free from interference during measurement are shown here.

To confirm the simulated beam pattern of the feed - reflector system, the telescope was swept in elevation and azimuth cuts over the sun. Corrections for the sun solid angle $\left(0.5^{\circ}\right)$, the aperture efficiency and elevation axis deformation was applied accordingly. In Fig. 3 the comparison between simulated and measured main beam is shown. At $418 \mathrm{MHz}$ there is compression of the beam due to saturation of the signal chain, this was corrected for at higher frequencies and the result is improved. The cuts were measured at a mean elevation of $43^{\circ}$ with a $\pm 8^{\circ}$ azimuth sweep (not to be confused with beam pattern azimuth coordinate $\phi$ in Fig. 3) over the sun with power averaged over the spectral channels, the bandwidth is $50 \mathrm{MHz}$.
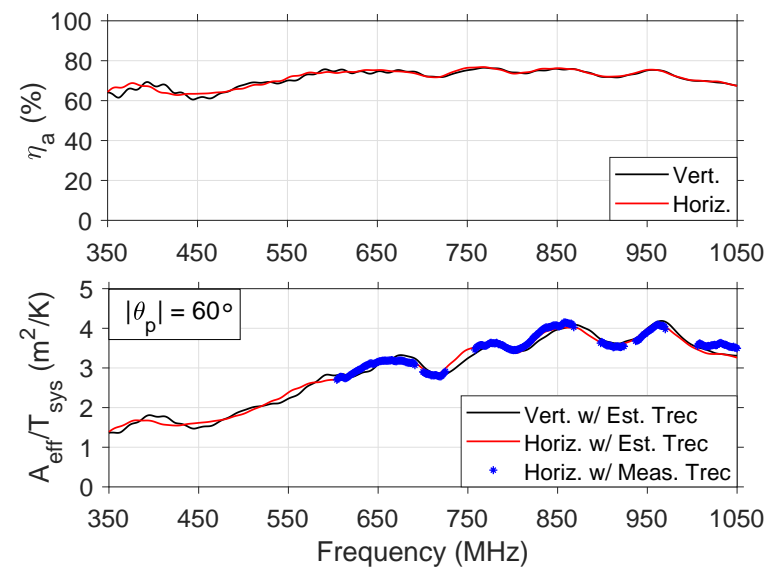

Fig. 2. Simulated aperture efficiency $\eta_{\mathrm{a}}$ (top) and sensitivity $A_{\text {eff }} / T_{\text {sys }}$ (bot.) for the feed on DVA-1 for both polarizations at zenith angle $\left|\theta_{p}\right|=60^{\circ}$. Sensitivity with measured $T_{r e c}$ is shown as blue dots.

\section{CONCLUSION}

A prototype room temperature single pixel feed system for SKA Band 1 over $350-1050 \mathrm{MHz}$ was installed and measured on the DVA-1 telescope. The beam pattern cuts measured by sweeping the sun gives a reasonable agreement with predicted beam cuts and the measured $T_{\text {rec }}$ proves the feasibility of the system with good agreement to predicted performance.

\section{ACKNOWLEDGEMENT}

This work has been funded by Swedish VR Research Infrastructures Planning Grant Swedish contributions to the SKA radio-telescope in its pre-construction phase. System simulator was provided by M. Ivashina at the Dept. of Electrical Eng., Chalmers University. The initial QRFH model was provided by I. Theron and R. Lehmensiek, EMSS Antennas, South Africa.

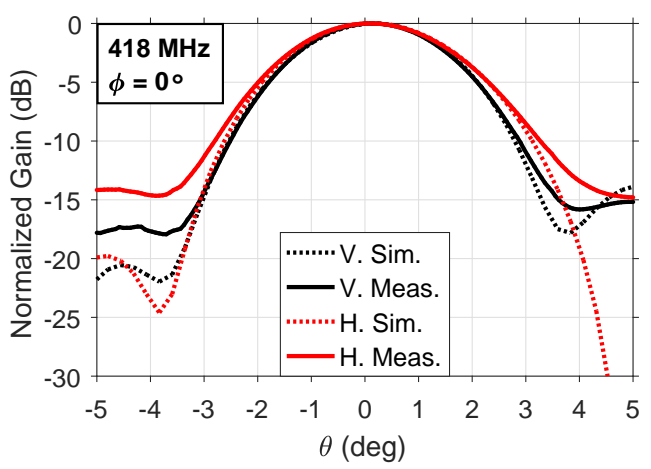

(a)

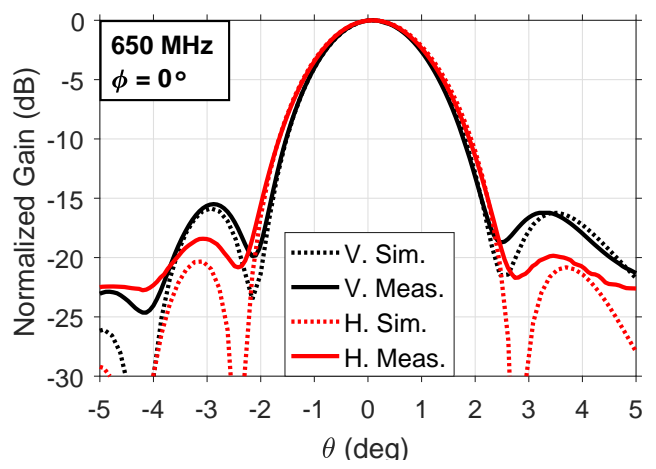

(b)

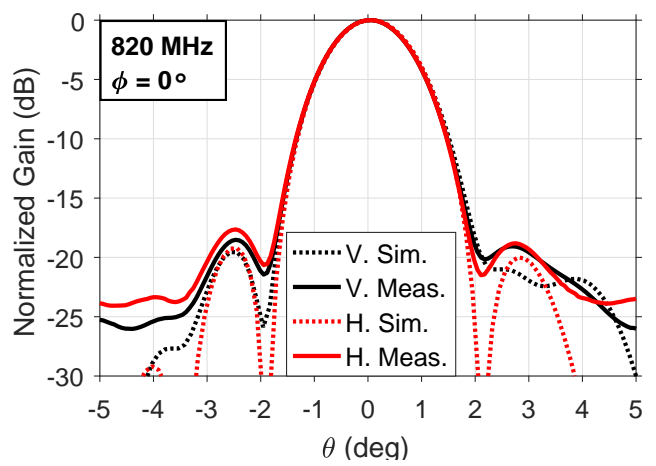

(c)

Fig. 3. Measured beam pattern compared to simulated for $\phi=0^{\circ}$ on DVA-1, normalized to $0 \mathrm{~dB}$, for both polarizations (V. for Vertical, H. for Horizontal). The sun was used as source and corrections for $\eta_{a}$, elev. axis deformation and the sun solid angle was applied. (a) $418 \mathrm{MHz}$ (b) $650 \mathrm{MHz}$ (c) $820 \mathrm{MHz}$.

\section{REFERENCES}

[1] B. Billade, J. Flygare, M. Dahlgren, B. Wästberg, and M. Pantaleev, "A wide-band feed system for SKA Band 1 covering frequencies from 350 $1050 \mathrm{MHz}$," in $10^{\text {th }}$ European Conference on Antennas and Propagation (EuCAP), Davos, Switzerland, April 2016, pp. 1-3.

[2] J. Schleeh, N. Wadefalk, P. A. Nilsson, and J. Grahn, "10 K room temperature LNA for SKA Band 1," in IEEE MTT-S Int. Microwave Symp. Dig. (IMS), San Francisco, CA, United States, May 2016, pp. 1-4.

[3] J. Flygare, B. Billade, M. Dahlgren, B. Wästberg, and M. Pantaleev, "Integrated calibration noise coupler for room temperature SKA Band 1 feed system," in IEEE International Symposium on Antennas and Propagation (APSURSI), San Diego, CA, USA, July 2017, pp. 1-2.

[4] M. Ivashina, O. Iupikov, R. Maaskant, W. van Cappellen, and T. Oosterloo, "An optimal beamforming strategy for wide-field surveys with phased-array-fed reflector antennas," IEEE Transactions on Antennas and Propagation, vol. 59, no. 6, pp. 1864-1875, June 2011. 